\title{
DYNAMIC NON-MINIMUM PHASE COMPENSATION FOR SISO NONLINEAR SYSTEMS
}

\author{
M. Bacic*, M. Cannon*, and B. Kouvaritakis* \\ *Oxford University, Department of Engineering Science, \\ Parks Rd, Oxford OX1 3PJ, UK,marko.bacic@eng.ox.ac.uk
}

\begin{abstract}
Nonminimum phase difficulties prevent the use of input-output feedback linearization. To avoid this problem, earlier work proposed the use of synthetic outputs with the view of placing zeros in prescribed locations under a condition of static equivalence. The objective here is also to introduce a synthetic output. However this is defined through the use of dynamic perturbations aimed at yielding maximum relative degree. Resetting of the initial conditions of the additional dynamics allows design freedom for closing the gap between the behaviour of the synthetic and actual output. The efficacy of the strategy in meeting pole placement is demonstrated by a simple numerical example. Copyright (C) 2005 IFAC
\end{abstract}

Keywords: non-minimum phase systems, feedback linearization, nonlinear control

\section{INTRODUCTION}

Unconstrained optimal control is of interest in its own right, but it also has found extensive use in the definition of terminal control laws deployed in model predictive control (Mayne et al, 2000). However the determination of the optimal is a rather challenging. An exception to this relates to the case when the optimal control cost does not include the usual term which places a penalty on control activity. Under such circumstances, Input-Output feedback linearization (Isidori, 1989) provides a convenient means for the explicit determination of the optimal control law. This results in minimum values for the cost, which typically (in the context of regulation) comprise the integral of the square of the system output. However there is no guarantee of stability in the presence of non-minimum phase characteristics. Roughly speaking, feedback linearization cancels the nonlinear dynamics from input to output thereby making the system internal dynamics unobservable from the system outputs. Thus in the case of unstable inverse dynamics, it is perfectly possible for the output to converge to zero but the state to diverge from the intended equilibrium point. These considerations apply in equal measure to model algorithmic control (Richalet et. al., 1978; Soroush and Kravaris,1992) where the (explicit or implicit) inversion of dynamics is aimed at achieving model reference, in place of striving for optimality.

This problem was tackled through non-minimum phase compensation and involves approximation. This is achieved through minimum/-non-minimum phase decomposition, or stable/anti-stable decomposition of the zero dynamics, or inner-outer approximate factorisations (Doyle et. al., 1992; Doyle et.al. 1996). Earlier work (Kravaris and Daoutidis, 1990) considered a particular canonical form which allows the derivation of ISE optimal solutions for the special case of 2nd order SISO systems. The extension of this work to higher order systems is not straightforward and instead later work suggested (Niemiec and Kravaris, 2001) the use of synthetic outputs which allow the definition of minimum phase systems. Such systems can be made to follow exactly a given reference signal and the design cycle then can be completed through a minimization of the deviation of the behaviour of the 
synthetic from the actual output. Further work on synthetic outputs followed (Martin et. al., 1996 and Fliess et. al., 1998).

The work here also makes use of synthetic outputs but rather than create those through a redefinition of the output map, the idea of introducing additional dynamics is proposed. Also, rather than place the system zeros from input to synthetic output at prescribed locations, the added dynamics are adjusted so that the system has no zeros at all. Moreover the minimization of the deviation of the behaviour of the synthetic from the actual output can be achieved through resetting the initial conditions of the added dynamics. The main aim is to explore the underlying philosophy and to show its viability in deriving closed loop stability while at the same time meeting some performance specification. For illustration purposes we adopt a pole placing objective; optimal control without a penalty on control activity and without constraints becomes a problem in "cheap control" which is of little practical use. The state space augmentation with the view of achieving maximum relative degree leads to partial differential equations which may be difficult to solve explicitly. Like the work presented in (Niemiec and Kravaris, 2001), at first sight it appears that our strategy requires explicit solution of equations which can be accomplished only in special second order cases. However our zero cancellation (and pole placement) can be implemented in a point-wise fashion so that extension to higher dimensions should be perfectly possible. Needless to say that the algebra gets progressively more cumbersome as the order increases however here for clarity the development of the paper is restricted to SISO 2nd order systems.

Reduction of the ideas of the paper to the linear case leads to the paradox that one should be able to achieve nearly zero cost under cheap control, even for systems with non-minimum phase zeros. All one has to do, is add extra dynamics to get rid of the transmission zeros and apply cheap control using the synthetic output. Given that under cheap control all the states (of the augmented system) can be made to decay arbitrarily fast it is then easy to show that the deviation between synthetic and actual outputs would also decay arbitrarily fast thereby indicating that synthetic and actual cost could be made to be arbitrarily small. This paradox would suggest that one can do better than optimal control, and whereas in some sense this is true, it is a theoretical artifice in that it depends on exact knowledge of the system parameters and is in fact arbitrarily sensitive to small parameter perturbations. This robustness issue however is the result of sensitivity due to the use of cheap control rather than due to the strategy of augmenting the system dynamics. Although robustness is not tackled explicitly in our paper, sensitivity tests show that our results enjoy a significant measure of robustness. No doubt, in the absence of input constraints, an unreasonable pole placement objective could well introduce robustness problems, but in part that could be counteracted (albeit at some sub-optimality) by the convergence conditions imposed on the resetting process.

\section{STATE SPACE AUGMENTATION FOR ZERO CANCELLATION}

Consider the second order affine in the input nonlinear model

$$
\dot{x}=f(x)+g(x) u, \quad y=C x
$$

where $x \in \mathbb{R}^{2}, u \in R, y \in R$ and for convenience the output is assumed to depend linearly on the states; if this is not the case, the algebra presented in the sequel is still possible but more involved. It is assumed that $x=0, u=0$ defines an equilibrium point. If this system were minimum phase about the origin, then input-output feedback linearization (IOFL) could be used to steer the state to the origin and to ensure that the output decays to zero according to the dynamics of a linear system with prescribed poles, e.g. two poles at say -1 . For brevity, we shall refer to this as pole-placement (PP). The challenge is to achieve PP when the inverse dynamics about the origin exist, i.e. when

$$
C g(x) \neq 0
$$

and when the zeros of the linearization of (1) about the origin lie in the right half of the complex plane. PP implies the need for IOFL but in the presence of nonminimum characteristics this may lead to instability; the inverse dynamics will be made unobservable from the output so that the output could be converging to 0 while the state diverges.

To overcome this, the proposal here is to introduce an extra state, $Z$, and synthetic output $\psi$ :

$$
\dot{z}=p(x)+q(x) u, \quad \psi=C x+z
$$

Due to the presence of non-minimum phase characteristics, it is not possible to attain exact PP from $u$ to $y$, however one could achieve this approximately by aiming for exact PP from $u$ to $\psi$ while seeking to minimize $Z$. The idea is to design $p(x), q(x)$ such that (1), (5) has maximum relative degree thereby enabling the application of IOFL. Accordingly, it is required that $\dot{\psi}, \ddot{\psi}$ are made independent of $u$ whereas $u$ is so chosen that $\dddot{\psi}$ is an appropriate linear combination of $\psi, \dot{\psi}, \ddot{\psi}$, e.g.

$$
\dddot{\psi}=-\psi-3 \dot{\psi}-3 \ddot{\psi}
$$

Let $n^{T}(x)$ be a vector function such that

$$
n^{T}(x) g(x)=0
$$


and such that the matrix of first order partial derivatives of $n^{T}(x)-C f_{x}(x)$ is symmetric:

$$
\frac{\partial\left[n-C f_{x}(x)\right]_{1}}{\partial x_{2}}=\frac{\partial\left[n-C f_{x}(x)\right]_{2}}{\partial x_{1}}
$$

Let also $\Gamma$ be a region containing the origin such that for all $x \in \Gamma$ the following holds true:

$$
g^{T}(x) n_{x}^{T}(x) f(x)+n^{T}(x) f_{x}(x) g(x) \neq 0
$$

where $n_{x}(x), f_{x}(x)$ denote the matrices of first order partial derivatives of the elements of $n(x), f(x)$, respectively with respect to $x_{1}, x_{2}$. Then it is possible to state the following results.

Theorem 2.1 For all $x \in \Gamma$ (1), (4) has maximum relative degree if and only if $p(x), q(x)$ satisfy:

$$
q(x)=-C f(x), p_{x}(x)=-C f_{x}(x)+n^{T}(x)
$$

Proof: Differentiation of (5) with respect to time and use of (1) and (4) gives

$$
\dot{\psi}=C \dot{x}+\dot{z}=C f(x)+p(x)+[C g(x)+q(z)] u
$$

which is independent from $u$ if and only if

$$
q(x)=-C f(x)
$$

For this choice of $q(x)$

$$
\dot{\psi}=C f(x)+p(x)
$$

Further differentiation and use of (1) gives

$$
\begin{aligned}
& \ddot{\psi}=C f_{x}(x) \dot{x}+p_{x}(x) \dot{x}=C f_{x}(x) f(x)+ \\
& +p_{x}(x) f(x)+\left[C f_{x}(x)+p_{x}(x)\right] g(x) u
\end{aligned}
$$

where $p_{x}^{T}(x)$ denotes the transpose of the gradient of $p(x)$. Then the condition

$$
\left[C f_{x}(x)+p_{x}(x)\right] g(x)=0
$$

is necessary and sufficient to make $\ddot{\psi}$ independent of $u$ :

$$
\ddot{\psi}=C\left[f_{x}(x)+p_{x}(x)\right] f(x)
$$

Eqn. (16) together with the definition of $n(x)$ suggest (11). Substitution of (11) into (15) gives:

$$
\ddot{\psi}=n^{T}(x) f(x)
$$

Differentiation of the above yields

$$
\dddot{\psi}=\dot{x}^{T} n_{x}^{T}(x) f(x)+n^{T}(x) f_{x}(x) \dot{x}
$$

so that after substitution into the above of (1) gives

$$
\begin{aligned}
& \dddot{\psi}=\left[\left(f^{T}(x)+g^{T}(x) u\right) n_{x}^{T}(x) f(x)+\ldots\right. \\
& +n^{T}(x) f_{x}(x)(f(x)+g(x) u)
\end{aligned}
$$

For $x \in \Gamma$ the above establishes that the third derivative of $\psi$ is the lowest derivative that does actually depend on $u$. This implies that the relative degree of (1), (4) is three, which for the assumed dimensions of (1) is the maximum it can be.

Corollary 2.2 For the $p(x), q(x)$ of Theorem 2.1 and for $x \in \Gamma$, the control law

$u=\frac{-C x-z-3(C f(x)+p(x))-3 n^{T}(x) f(x)-f^{T}(x) n_{x}^{T}(x) f(x)}{g^{T}(x) n_{x}^{T}(x) f(x)+n^{T}(x) f_{x}(x) g(x)}$

$-\frac{f^{T}(x) n_{x}^{T}(x) f(x)+n^{T}(x) f_{x}(x) f(x)}{g^{T}(x) n_{x}^{T}(x) f(x)+n^{T}(x) f_{x}(x) g(x)}$

ensures satisfaction of the PP condition (6).

Proof: For all $x \in \Gamma$ the control law of (21) is defined. The remainder follows by a straight forward substitution of (14), (18) and (21) into (6)

\section{POINTWISE SOLUTION OF PP CONDITIONS}

The results of $\$ 2$ require the computation of $n(x)$, and although both (7) and (8) seem straightforward, in general the solution for $n(x)$ can be challenging. The reason for this is that whereas from (7) it follows that direction of $n^{T}(x)$ is fixed and must be that of the left null space of $g(x)$, the scaling factor has to be such that it satisfies (8). This implies the need for solution of a partial differential equation which can be solved, using the method of characteristics, but which in general is rather challenging. This can be avoided by forcing the scaling to be 1 along the trajectories of (1), (4) and by treating any given $X$ as a constant but ensuring that $n^{T}(x)$ and $p(x)$ satisfy at the given $x$ all the differential properties implied by the conditions of Theorem 2.1. Thus at a given $x, n^{T}(x)$ can be taken to be the unit vector in the direction of the left null space of $g(x)$, but since (7) must be identically true, we must also have that

$$
n_{x}^{T} g+g_{x}^{T} n=0
$$

where the argument "(x)" has been purposely suppressed (since the given $X$ is now treated as a constant). The above gives the solution for $n_{x}^{T}$ as 


$$
n_{x}^{T}=-\frac{1}{g^{T} g} g_{x}^{T} n g^{T}+\left[\begin{array}{l}
\gamma_{1} n^{T} \\
\gamma_{2} n^{T}
\end{array}\right]
$$

However the off diagonal elements of $n_{x}^{T}$ must also satisfy (8) at $X$ so that

$$
\begin{aligned}
& -\frac{e_{1}^{T} g_{x}^{T} n g^{T} e_{2}}{g^{T} g}+\gamma_{1} n^{T} e_{2}-\frac{\partial\left[C f_{x}\right]_{2}}{\partial x_{1}}= \\
& =-\frac{e_{2}^{T} g_{x}^{T} n g^{T} e_{1}}{g^{T} g}+\gamma_{2} n^{T} e_{1}-\frac{\partial\left[C f_{x}\right]_{1}}{\partial x_{2}}
\end{aligned}
$$

which implies that $\gamma_{2}$ and hence $n_{x}^{T}$ can be expressed as affine functions of $\gamma_{1}$ :

$$
\gamma_{2}=l\left(x_{1}\right), \quad n_{x}^{T}=L\left(\gamma_{1}\right)
$$

On the other hand, forcing the norm of $n(x)$ to be 1 along the trajectories of (1), it follows that at any given $x$ we must have $\dot{n}^{T} n=0$ which implies that

$$
\dot{x}^{T} n_{x}^{T} n=\left[f^{T}+u g^{T}\right] n_{x}^{T} n=0
$$

The combination of (26) with (25) yields

$$
u=-\frac{f^{T} L\left(\gamma_{1}\right) n}{g^{T} L\left(\gamma_{1}\right) n}
$$

Theorem 3.1 At each $x \in \Gamma$, for $\gamma_{1}$ such that

$$
\begin{aligned}
& \left(C x+z+3(C f+p)+3 n^{T} f+f^{T} n_{x}^{T} f+n^{T} f_{x} f\right) g^{T} L\left(\gamma_{1}\right) n \\
& =\left(g^{T} n_{x}^{T} f+n^{T} f_{x} g\right) f^{T} L\left(\gamma_{1}\right) n
\end{aligned}
$$

the control law

$$
u=\frac{-C x-z-3(C f+p)-3 n^{T} f-f^{T} L\left(\gamma_{1}\right) f-n^{T} f_{x} f}{g^{T} L\left(\gamma_{1}\right) f+n^{T} f_{x} g}
$$

ensures that (at the given $X$ ) PP of (6) is satisfied.

Proof: Equation (28) is the consequence of the $u$ of Corollary 2.2 which ensures the satisfaction of (6) also satisfying the necessary condition of (27). Furthermore (29) is just (27) re-written for the solution of $n_{x}^{T}$ that satisfies the necessary conditions (8) and (22), namely for the $n_{x}^{T}$ as given by (25).

Although the expression in Theorem 3.1 and the relevant results may appear somewhat involved, they are all amenable to a straightforward computer implementation. Thus, (28) is a quadratic for which solutions can be computed explicitly, whereas the values of $Z$ required in both (28) and (29) can be obtained through the use of an ordinary differential equation solver of (1), (4) for the $u$ of (29). The values of $p$ can also be obtained from a ODE solver because along the trajectories of (1), it follows that

$$
\dot{p}=p_{x}^{T} \dot{x}=p_{x}^{T}(f(x)+g(x) u)
$$

The pointwise treatment of this section avoids the need for explicit expressions for $p$ and can be applied to any $2^{\text {nd }}$ order affine in the input system. More importantly it can be extended to higher dimensions, although then both $p(x), q(x)$ must become vector functions; augmentation by a single extra state $Z$ would no longer provide enough degrees of freedom for the achievement of maximum relative degree. The detailed treatment of the general dimension case forms the object of future research.

\section{THE ONLINE ALGORITHM AND ITS CONVERGENCE}

The attainment of maximum relative degree implies that the control law of Theorem 3.1 is not only stabilizing in $w$-space, where $w=\left[\begin{array}{lll}\psi & \dot{\psi} & \ddot{\psi}\end{array}\right]^{T}$, but also in $V$-space, where $V=\left[\begin{array}{ll}x^{T} & Z\end{array}\right]^{T}$; the danger of unstable inverse dynamics which are unobservable unstable dynamics no longer exists. Such a scheme would be fine if the objective were to drive $w$ to zero while $\psi$ observes the linear dynamics of (6). However the concern here is to cause $v$ to converge to the origin while $y$ decays according to the linear dynamics of (6). The difference between the synthetic and actual output is given by $Z$ and therefore the minimization of $Z$ forms a sensible design objective. However $Z$ is an artificial state introduced to achieve maximum relative degree and this is achieved by observing the differential conditions of Sections 2 and 3. Therefore one is free to reset the initial condition for $Z$ back to zero at each instant when the control $u$ of Theorem 3.1 is computed. Clearly the same applies to $p$ as well and resetting takes place at a high frequency, then given that $w$ is constrained to converge to the origin, it follows that both $Z$ and $p$ will stay close to zero. Of course it is known that $Z$ and $p$ cannot remain at exactly zero all the time because then the control of Theorem 3.1 would be de-stabilizing (on account of the assumed non-minimum phase characteristics of (1), (2)). In essence therefore the suggested scheme of resetting $Z$ and $p$ allows for small perturbations on the IOFL control law which cause it to become stabilizing. However, whereas without resetting, stability was guaranteed, it now becomes necessary to invoke a convergence condition.

To establish the convergence condition, consider the case without resetting for which the dynamics of (6) can be described in state space form as 


$$
\dot{w}=A w, \quad A=\left[\begin{array}{ccc}
0 & 1 & 0 \\
0 & 0 & 1 \\
-1 & -3 & -3
\end{array}\right]
$$

However $z, p$ would evolve as per (4) and (30) and could therefore grow to be sufficiently large so that the behaviour of the synthetic and actual output could be very different. The question now arises as to whether every $h$ seconds, where $h$ is an arbitrarily small time interval, one could perturb the values of $z, p$ by $\Delta z, \Delta p$ thereby also perturbing the values of $\psi, \dot{\psi}$, the first and second elements of $W$, the first of which is directly proportional to $Z$ and the other directly proportional to $p$, with the constants of proportionality both being equal to 1 (see equations (5) and (14)). The consequence of this would be to cause the behaviour of $w$ to deviate away from that predicted by (31). To ensure that $w$ still converges (albeit not necessarily as fast as indicated by (31)), it becomes necessary to restrict the size of the perturbation on $w$.

$$
\delta=\left[\begin{array}{lll}
\Delta z^{T} & \Delta p^{T} & 0
\end{array}\right]^{T}
$$

Theorem 4.1 Let $\mathrm{Q}$ be the solution of

$$
A^{T} Q+Q A=-\eta e_{1} e_{1}^{T}, \quad e_{1}=\left[\begin{array}{lll}
1 & 0 & 0
\end{array}\right]^{T}
$$

Then the law of Th3.1 with resetting constrained by

$$
(w+\delta)^{T}\left[A^{T} Q+Q A\right](w+\delta) \leq-\eta w^{T} e_{1} e_{1}^{T} w
$$

will cause $w$ to converge to 0 and guarantees that

$$
\int_{0}^{\infty} \psi^{2}(t) d t \leq \frac{1}{\eta} w_{o}^{T} Q w_{o}
$$

where $w_{o}$ denotes the initial value of $w$.

Proof: The conditions of Theorem 3.1 ensure that between resetting the dynamics of $w$ will be dictated by (31). (34) ensures that $V=w^{T} Q w$ is a Lyapunov function which at all times satisfies

$$
\dot{V} \leq-\eta \psi^{2}
$$

Condition (33) ensures that at all times there exist $\delta$ that satisfy (34), with $\delta=0$ being one of them. As a consequence $v$ will decrease monotonically so that upon integration of (36) one gets the result of (35).

Remark 4.1 In practice $h$ may be chosen not to be arbitrarily small, and the analysis of Theorem 4.1 could be applied to the linear discrete time dynamics implied by (31). However this is a straightforward technicality which will not be considered further.

Remark 4.2 The upper bound on the $l_{2}$-norm of $\psi$ depends on both $\eta$ and $Q$ so that in order to make this tight for general initial conditions one could minimize (offline) the trace or determinant of $Q$ for any particular choice of $\eta$ subject to condition (33) with the equality sign replaced by " $\leq$ ". The resulting minimization can be shown to be a convex optimisation for which there exist efficient solvers.

Algorithm 4.1 Implement the control law of Theorem 3.1 with $Z=0, p=0$ if the required perturbation $\delta$ satisfies (34) otherwise select the $\delta$ which satisfies (34) and minimizes $z^{2}+p^{2}$.

Remark 4.3 For small $h$ the change in $z, p$ between resetting times will be small so that starting from $z=0, p=0$ the $\delta$ required to reset $z, p$ at zero will be small and therefore for sufficiently small $\eta$ such $\delta$ are likely to be feasible (i.e. to satisfy (34)). Under such circumstances the minimization of Algorithm 4.1 will not be required and both $Z$ and $p$ will be small thereby suggesting a near exact satisfaction of the PP objective. If the $\delta$ above are infeasible, it is pointed out that the minimization of the Algorithm is trivial as it involves finding the point of tangency between an ellipse (defined by (34)) and a circle (corresponding to the equation $z^{2}+p^{2}=r^{2}$ ). This would be true for extensions to higher dimensions, except of course that the ellipses would become ellipsoids and the circles spheroids.

\section{ILLUSTRATIVE EXAMPLE}

For simplicity consider the bilinear system

$$
\begin{aligned}
\dot{x}=A x+(B+F x) u, \quad y=C x \\
A=\left[\begin{array}{cc}
-.72 & -1.18 \\
-.78 & -.59
\end{array}\right], B=\left[\begin{array}{l}
-5.2370 \\
-8.5732
\end{array}\right], C=\left[\begin{array}{ll}
-.69 & .2
\end{array}\right] \\
F=\left[\begin{array}{cc}
.6892 & -.4610 \\
.7056 & -1
\end{array}\right]
\end{aligned}
$$

This example was chosen to be challenging in that the origin is unstable both for $u=0$ and for IOFL. For this example the condition

$$
g^{T}(x) n_{x}^{T}(x) f(x)+n^{T}(x) f_{x}(x) g(x)=0
$$

define the two lines labelled as "abc" and "def" in Figure 1. They divide the $x$-plane into four quadrant of which the one containing the origin is $\Gamma$. The figure shows the closed loop trajectories generated by Algorithm 4.1 for a variety of initial conditions. It is pointed out that for the chosen initial values, the nonlinear effect of the term " $F X$ " is significant. The convergence parameter was chosen $\eta=10^{-3}$ and the associated minimum determinant $Q$ (see Remark 4.2) was found to be

$$
Q=\left[\begin{array}{lll}
.0021 & .0017 & .0005 \\
.0017 & .0018 & .0006 \\
.0005 & .0006 & .0002
\end{array}\right]
$$


The crosses on the figure mark the instances when (34) became active thereby preventing a resetting to zero of $z$ and $p$. The efficacy of Algorithm 4.1 is illustrated by the plots of Figure 2. Plot (a) depicts the behaviour of $y$, whereas (b) shows the evolution of $Z$. The very small values of $Z$ indicate that for those release conditions the algorithms achieved near exact PP. The plots of Figure 3 replicate the results of Figure 2 for the case when the model of equation (37) is used in the computation of the control law but the elements of the matrices are made subject to random time varying additive perturbations of up to $10 \%$. Algorithm 4.1 can be seen to enjoy a high degree of robustness.

\section{CONCLUSIONS}

Exact IOFL is destabilizing around non-minimum phase equilibrium points. This paper proposed a scheme which removed this difficulty by applying IOFL to a synthetic output which is created through a dynamic perturbation of the actual output. The perturbation was design to achieve maximum relative degree but otherwise was kept as small as possible thereby minimizing the deviation of synthetic from actual output. The development was presented in the context of second order systems but it is believed that extensions to the general case are possible and form the focus of future research. The ability to adjust the control law online also fosters the possibility of extensions which accommodate input constraints. Simulations show that for the chosen illustrative example our algorithm enjoys a high degree of robustness but a systematic study of this issue still remains to be completed.

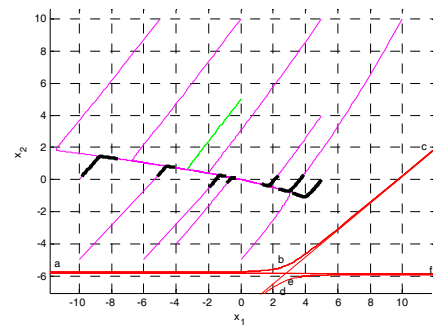

Fig. 1. State trajectories

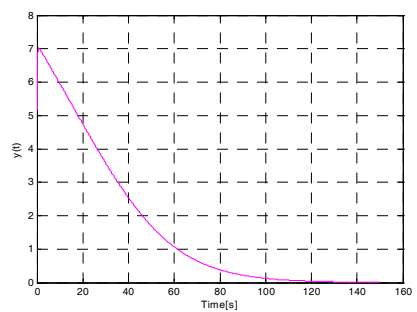

Fig. 2(a). Output trajectory(perfect model)

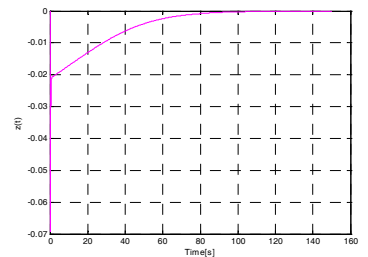

Fig. 2(b). z trajectory(perfect model)

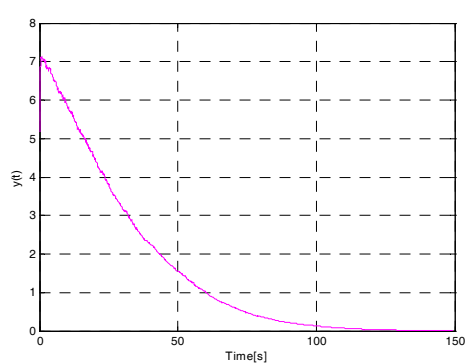

Fig. 3(a). Output trajectory (random model error)

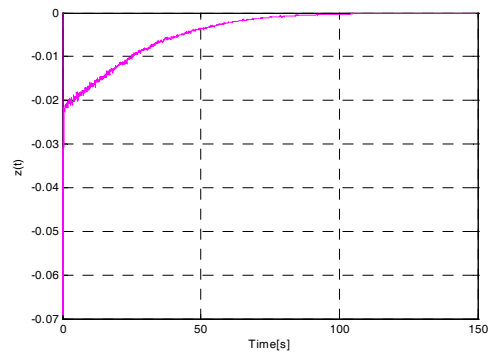

Fig. 3(b). $\mathrm{z}$ trajectory (random model error)

\section{REFERENCES}

Doyle, F. J. III, Allgower, F. and Morari, M. (1996). A normal form approach to approximate inputoutput linearization for maximum phase nonlinear SISO systems, IEEE Trans. AC, 41, 305-309

Doyle, F.J. III, Allgower, F., Oliveira, S., Gilles E. and Morari, M.(1992). On nonlinear systems with poorly behaved zero dynamics, Proceedings of ACC, 1992, 2571-2575

Fliess, M., Sira-Ramirez, H. and Marquez, R. Regulation of non-minimum phase outputs: a flatness based approach. Perspectives in Control - Theory and Applications: a Tribute to Ioan Dore Landau, 143--164

Isidori, A. (1989). Nonlinear Control Systems: An Introduction, Springer Verlag.

Kravaris, C. and Daoutidis, P. (1990). Nonlinear state feedback control of second-order nonminimumphase nonlinear systems, Comnputers Chem. Engng, 14, No. 4/5, 439-449

Martin, P., Devasia, S. and Paden, B. A different look at output tracking: Control of a VTOL aircraft, Automatica, 36,101-107

Mayne, D.Q., Rawlings, J.B., Rao, C.V. and Scokaert, P. O. M. (2000). Constrained model predictive control: stability and optimality, Automatica, 36, 789-814

Niemiec, M. and Kravaris, C. (2001). Nonlinear model-algorithm control for multivariable nonminimum-phase process, in B Kouvaritakis and $\mathrm{M}$ Cannon (eds.) Nonlinear predictive control: theory and practice, IEE, London

Richalet, J., Rault, A., Testud, J.L. and Papon, J. (1978). Model predictive heuristic control: application to industrial processes, Automatica, $14,413-428$

Soroush, M. and Kravaris, C. (1992). Discrete-time nonlinear controller synthesis by input/output linearizatino, AIChE J, 38, 12, 1923-1934 